\title{
Testing the Efficacy of Bio Control Agents and Fungicides against Fusarium oxysporum f. sp. ciceris under in vitro Conditions
}

\author{
P. Murali Sankar ${ }^{1 *}$, S. Vanitha ${ }^{1}$, A. Kamalakannan ${ }^{1}$, P. Anantha Raju ${ }^{2}$, \\ P. Jeyakumar ${ }^{3}$ and T. Raguchander ${ }^{1}$ \\ ${ }^{1}$ Department of Plant Pathology, ${ }^{2}$ Department of Pulses, ${ }^{3}$ Department of Crop Physiology, \\ Tamil Nadu Agricultural University, Coimbatore-641003 India \\ *Corresponding author
}

A B S T R A C T

\begin{tabular}{|l|}
\hline K e y w or d s \\
Rhizobacteria, \\
$\begin{array}{l}\text { Pseudomonas sp., } \\
\text { Carbendazim, } \\
\text { Siderophore and CPs3 }\end{array}$ \\
\hline Article Info \\
\hline $\begin{array}{l}\text { Accepted: } \\
\text { 02 May 2018 } \\
\text { Available Online: } \\
\text { 10 June } 2018\end{array}$ \\
\hline
\end{tabular}

\section{Introduction}

Chickpea (Cicer arietinum L.) is an important legume crop in the world for its easy available form of edible proteins and vitamins. In India it is cultivated at cool winter (Rabi) season in semi-arid tropics by irrigated or rain-fed conditions (Nene et al., 1984). India is largest producer of chickpea in world sharing 65.25 per cent in area and 65.49 per cent in production and is grown on 10.23 million ha area with production 9.88 million tonnes and
Fusarium oxysporum $\mathrm{f}$. sp. ciceris is a ubiquitous seed and soil borne pathogen in several crops. It survives in the soil without host from several years. To manage the wilt pathogen is such as a difficult in crop management. The rhizobacteria as grouped under two categories by the cultural, morphological and biochemical characters. Ten isolates (CPs1 to CPs9) were identified as Pseudomonas sp. by medium to strong production of $\mathrm{KOH}$, $\mathrm{HCN}$ production and siderophore synthesis. Another ten isolates of (CBs1 to CBs10) identified as brown creamish and serrated margins with production of catalase and citrate. Under in vitro efficacy, Pseudomonas isolates CPs3 showed maximum inhibition per cent of $54.07 \%$ followed Pf1 at $51.47 \%$. In Bacillus sp. isolate CBs5 showed maximum inhibition per cent of $61.85 \%$ and followed CBs1 recorded $59.25 \%$ and fungal antagonistic activity Tv1 showed maximum mycelial inhibition per cent of $67.77 \%$ followed by CTs 3 at $62.22 \%$. Carbendazim recorded maximum mycelial growth inhibition of $86.66 \%$.

productivity $967 \mathrm{~kg} / \mathrm{ha}$ (Thaware et al., 2017). Despite the production was reduced due to several biotic and abiotic factors. Chickpea is noticed to be more than 52 pathogens at cropping season (Nene et al., 1984). Among these pathogens $F$. oxysporum f. sp. ciceris causing a potential yield loss for both in seed yield and seed weight by wilt about 10 to 15 per cent (Navas-Cortes et al., 2000; Khilare et al., 2009). Although many control measures have been developed for management the wilt disease in chickpea, the soil borne nature of 
pathogen make control difficult. An extensive using of resistant cultivars, the pathogen races were breakdown the host resistance and probably attempted higher incidence throughout the world (Inam-Ul-Haq et al., 2015). But cultural and chemical practices provided significantly reduction in the disease incidence and it should change the crop diversity due to highly influence of soil conservation by usage of chemicals (Nene and Reddy, 1987).

Out of these, several measures biocontrol agents were reported to an alternative potential tool for management of pathogens (ElKatatany et al., 2003). Because, the crop rhizosphere holding Plant growth-promoting rhizobacteria (PGPR) is the initial barrier for invasion of soil borne pathogens attack (Weller, 1988; Joseph et al., 2007). The Plant growth promoting rhizobacteria (PGPR) improve the plant growth by root colonization and derived a resistance against soil borne pathogens through initiate strongly nutrition competition, production of antibiotics, N2 fixation, extracellular hydrolytic enzymes, secondary metabolites such as hydrogen cyanide and induced systemic resistance (Khan et al., 2009; Datta et al., 2011). A large array of bacteria including species of Pseudomonas, Azospirillum, Azotobacter (Ahmad et al., 2008), Bacillus, Beijerinckia, Klebsiella and Serratia (Gyaneshwer et al., 2001) have been reported to widely adapted soil antagonistic species for eco-friendly without harmful for respected host also (Kamala Devi, 2012). It is beneficial to wide crop diversity in agriculture production (Govindarajan et al., 2006). These biocontrol agents were mostly depended on the host and soil ecosystem (Hervas et al., 1997).

Rhizobacteria group of Bacillus and $P$. chlororaphis strongly antagonistic against with three races $(0,1$ and 5$)$ of $F$. oxysporum f. sp. ciceris by their antibiotics synthesis under in vitro conditions and suppressed the utilization of chemicals in field conditions also (Landa et al., 1997). In fungal antagonist, Trichoderma, Gliocladium, Candida, Ampelomyces, Coniothyrium placed in potential role against several plant pathogens (Liu et al., 1995). Trichodrma is a secondary opportunistic invader and colonized in rhizobiome and changes plant metabolism by nutrient uptake from soil, plant growth and increase primary defense against pathogen invasion (Poddar et al., 2004). Several species Trichoderma were notified strong antagonistic activity against soil borne pathogens viz., $F$. oxysporum, $F$. solani, $M$. phaseolina and $S$. rolfsii, by their generation of ROS, lytic enzymes and secondary metabolites (Singh et al., 2009). Hossain et al., (2013) reported that T. harzianum (T75) isolate completely inhibited the mycelial proliferation of $F$. oxysporum f. sp. ciceris in dual culture assay. Although each of these practices individually potential, yet none is completely viable when applied alone the problem still throughout the world. The chemical based control is most effective and reliable. No economical and ecofriendly strategies are available to combat this devasting soilborne pathogen. So, to using new chemical fungicides potential for control new races of pathogens management (Sharma et al., 2010).

The present study was carried out for evaluate the bioefficacy of biocontrol agents from chickpea rhizosphere and fungicides against with $F$. oxysporum f. sp. ciceris under in vitro.

\section{Materials and Methods}

\section{Isolation and identification of rhizobacteria}

Rhizosphere colonizing twenty bacterial isolates was isolated from different rhizosphere soils of chickpea from major growing areas of Tamil Nadu. The soil particles tightly adhered with root portion of 
chickpea were gently removed and suspended in $10 \mathrm{ml}$ sterile distilled water. After serial diluted (upto $10^{6}$ ) one $\mathrm{ml}$ of suspension from each $10^{3}$ to $10^{6}$ dilutions transferred into sterile Petri plates containing Nutrient agar media (Bacillus sp.) and King's B media (Pseudomonas sp.) for the isolation of respectively by kept under $30^{\circ} \mathrm{C}$ for 24 hours.

The further purification is done in the respective media and kept under laboratory conditions. The isolates were identified based on their phenotypical and morphological characters (Cakmakci et al., 2007).

\section{Biochemical characterization}

The bacterial isolates were biochemical characterized on the basis of $\mathrm{KOH}$ test, $\mathrm{HCN}$ production, gelatine hydrolysis, siderophore production, catalase production, starch hydrolysis; citrate utilization and influence of $\mathrm{NaCl}$ for growth were checked as per the standard methods for followed for Pseudomonas and Bacillus sp. The reactions were referred as (+) - Positive; (-) - Negative $(++)$ - Medium production; (+++) - Strong production (Schaad, 1992).

\section{Isolation and identification of Trichoderma sp.}

Soil samples were collected from chickpea rhizosphere of nine various locations. The soil particles were gently removed and suspended in $10 \mathrm{ml}$ sterile distilled water. After serial diluted $\left(10^{3}\right)$ one $\mathrm{ml}$ of suspension was transferred into sterile Petri plates containing Trichoderma selective media (Glucose - 3g, $\mathrm{K}_{2} \mathrm{HPO}_{4}-0.9 \mathrm{~g}, \mathrm{MgSO}_{4}-0.2 \mathrm{~g}, \mathrm{KCl}_{2}-0.5 \mathrm{~g}$, $\mathrm{NH}_{4} \mathrm{Cl}_{2}-1.05 \mathrm{~g}$, Rose Bengal - $0.15 \mathrm{~g}$, chloromphenical - 0.25g, metalaxyl - 0.3g, Agar - 15g, D. water - 1lit). After three days the green coloured colonies are sub cultured in PDA containing Petri plates and kept under $4^{\circ} \mathrm{C}$ for further studies (Thaware et al., 2017).

\section{In vitro efficacy of biocontrol agents}

Antagonistic activity of the ten bacterial isolates of Pseudomonas sp. (CPs1 - CPs9 and Pf1 as check), Bacillus sp. (CBs1-CBs10) and fungal antagonist for ten Trichoderma sp. (CTs1-CTs10) were against $F$. oxysporum $\mathrm{f}$. sp. ciceris isolate (Foc4) evaluated based on dual culture technique (Landa et al., 1997) and replicated thrice. Radial growth of the fungus was measured and percentage of growth inhibition was calculated using the formula:

Inhibition $(\%)=(\mathrm{R}-\mathrm{r}) / \mathrm{R} \times 100$

Where, $r$ is the radius of the fungal colony opposite the bacterial colony and $\mathrm{R}$, is the maximum radius of the fungal colony in the absence of the bacterial colony (Kumari and Khanna, 2014).

\section{In vitro efficacy of fungicides (Poisoned food technique)}

The efficacy of eight different following fungicides viz., Carbendazim (Bavistin), Ntrichloromethylthio-4-cyclohexane 1,2dicarboximide (Captan), Copperoxychloride (Kocide), Cymoxanil + Mancozeb (Curzate), Fenamidone + Mancozeb (Sectin), Fluopyram (Luna Experience), Iprovalicarb + Probieb (Melody Duo) and Isoprothiolane (Fujione) at different conc. of $0.025,0.05$ and $0.1 \%$ were tested on the radial mycelial growth of the pathogen by poisoned food technique (Kumar and Mane, 2017). At first, the stock solution of above fungicides at different concentration was prepared and the required concentration of fungicides was mixed with PDA medium in sterile conical flask. The three different concentrations of the fungicides were poured individually in sterile petridish at $20 \mathrm{ml}$ and allowed to solidify. Eight mm of mycelial disc of the pathogen (Foc 4) was placed in the centre and incubated at room temperature $\left(28 \pm 2^{\circ} \mathrm{C}\right)$. The three replications have been 
maintained for each concentration and untreated control was also maintained. The radial mycelial growth of the pathogen was observed at seven days after inoculation (Subhani et al., 2011; Maitlo et al., 2014).

Per cent inhibition over control $=\frac{\text { C }-\mathrm{T}}{\mathrm{C}}$

Where,

C- Mycelial growth of pathogen in control

$\mathrm{T}$ - Mycelial growth of pathogen treated plates

\section{Results and Discussion}

\section{Isolation and identification of rhizobacteria}

The different isolates of rhizobacteria were isolated by dilution method and it was grouped and characterized by their cultural and morphological, ten isolates of Pseudomonas sp. were phenotypically appeared as pale yellow to white in colour and translucent slimy and Bacillus species were showed pale brown to dull white in coloured, serrated and wavy margin (Table 1).

These results revealed that (Kumari and Khanna, 2014) isolated 40 rhizobacterial isolates from chickpea and 20 isolates were confirmed as Pseudomonas sp. and 16 isolates reported as Bacillus sp. from chickpea. Landa et al., (1997) reported that slimy and fluorescent growth of rhizobacteria was confirmed as seven isolates of Pseudomonas sp. from chickpea.

\section{Biochemical characterization rhizobacteria}

The isolated bacterial antagonists were biochemical characterized viz., ten isolates exhibited medium production in $\mathrm{KOH}$ test and gelatine hydrolysis. In $\mathrm{HCN}$ production and siderophore production isolates viz., Pf1, CPs3 and CPs9 were showed strong production and confirmed as Pseudomonas sp. Another ten isolates of rhizobacteria were showed medium production in catalase test, starch hydrolysis, citrate utilization and growth in $\mathrm{NaCl}$ and confirmed as Bacillus sp. (Table 2). Hundred and fifty isolates of PGPR from rhizosphere of chickpea among these isolates, 35 belonged to Pseudomonas sp. and 40 under Bacillus sp. by biochemical reaction of hydrogen cyanide production, siderophore synthesis, catalase and nitrate reduction, (Joseph et al., 2007). Karimi et al., (2012) reported that six Bacillus isolates exhibited strong production of IAA synthesis and protease.

\section{Isolation and identification of Trichoderma sp.}

The dilution plate method, after three days, green coloured mycelial antagonistic fungi (CTs1 to CTs9) was isolated from nine different locations and sub cultured in PDA contained Petri dishes and identified through all the isolates were produced cylindrical numerous conidia (Table 3 ).

These results revealed that Hossain et al., (2013) reported that 20 isolates of Trichoderma sp. (T-1 to T-77/2) were isolated and identified from various cultivars of chickpea.

\section{In vitro efficacy of biocontrol agents}

\section{Pseudomonas sp.}

Antagonistic activity among the 10 of Pseudomonas sp. against $F$. oxysporum f. sp. ciceris (Foc4) in dual culture assay, all the isolates showed significant inhibition per cent from $10.36 \%$ to $54.07 \%$. Out of these ten isolates, isolate CPs3 showed maximum inhibition per cent of $54.07 \%$ followed Pf1 at $51.47 \%$ (Table 4; Plate 1). 
Table.1 Phenotypic and morphological characterization of antagonistic rhizobacteria from chickpea

\begin{tabular}{|c|c|c|c|c|c|}
\hline $\begin{array}{l}\text { S. } \\
\text { No }\end{array}$ & Locations & $\begin{array}{l}\text { Isolate } \\
\text { code }\end{array}$ & $\begin{array}{l}\text { Phenotypic } \\
\text { characters }\end{array}$ & $\begin{array}{l}\text { Morphological } \\
\text { edge }\end{array}$ & Referred as \\
\hline 1. & Coimbatore & $\begin{array}{c}\text { Pf1 } \\
\text { (TNAU) }\end{array}$ & $\begin{array}{l}\text { Creamy, } \\
\text { translucent, slimy }\end{array}$ & Rounded & P. fluorescens \\
\hline 2. & $\begin{array}{l}\text { Gomangalam } \\
\text { pudur }\end{array}$ & CPs 1 & $\begin{array}{l}\text { Creamy, light } \\
\text { translucent, slimy }\end{array}$ & Rounded & $\begin{array}{c}\text { Pseudomonas } \\
\text { sp. }\end{array}$ \\
\hline 3. & Thippampatti & CPs2 & $\begin{array}{l}\text { Pale yellowish, } \\
\text { translucent, slimy }\end{array}$ & Rounded & $\begin{array}{c}\text { Pseudomonas } \\
\text { sp. }\end{array}$ \\
\hline 4. & Mukkonam & CPs3 & $\begin{array}{l}\text { Milky white, } \\
\text { translucent, slimy }\end{array}$ & Rounded & $\begin{array}{l}\text { Pseudomonas } \\
\text { sp. }\end{array}$ \\
\hline 5. & Modakkupatti & CPs4 & $\begin{array}{l}\text { Brownish, } \\
\text { translucent, slimy }\end{array}$ & Rounded & $\begin{array}{l}\text { Pseudomonas } \\
\text { sp. }\end{array}$ \\
\hline 6. & Valzavadi & CPs5 & $\begin{array}{l}\text { Pale white, } \\
\text { translucent, slimy }\end{array}$ & Rounded & $\begin{array}{c}\text { Pseudomonas } \\
\text { sp. }\end{array}$ \\
\hline 7. & $\begin{array}{l}\text { Ramachadra } \\
\text { Puram }\end{array}$ & CPs6 & $\begin{array}{l}\text { Creamy yellow, } \\
\text { translucent, slimy }\end{array}$ & Rounded & $\begin{array}{l}\text { Pseudomonas } \\
\text { sp. }\end{array}$ \\
\hline 8. & P.N.Palayam & CPs7 & $\begin{array}{l}\text { Creamy white, } \\
\text { translucent, slimy }\end{array}$ & Rounded & $\begin{array}{l}\text { Pseudomonas } \\
\text { sp. }\end{array}$ \\
\hline 9. & Poolankinaru & CPs8 & $\begin{array}{l}\text { Brownish, } \\
\text { translucent, slimy }\end{array}$ & Rounded & $\begin{array}{l}\text { Pseudomonas } \\
\text { sp. }\end{array}$ \\
\hline 10. & Konnur & CPs9 & $\begin{array}{l}\text { Pale white, } \\
\text { translucent, slimy }\end{array}$ & Rounded & $\begin{array}{c}\text { Pseudomonas } \\
\text { sp. }\end{array}$ \\
\hline 11. & Gomangalam & CBs1 & Pale brown slimy & $\begin{array}{l}\text { Serrated point } \\
\text { margin }\end{array}$ & Bacillus sp. \\
\hline 12. & Thippampatti & $\mathrm{CBs} 2$ & Pale brown slimy & $\begin{array}{l}\text { Thick serrated } \\
\text { margin }\end{array}$ & Bacillus sp. \\
\hline 13. & Mukkonam & CBs3 & Pale white slimy & $\begin{array}{l}\text { Wavy winged } \\
\text { margin }\end{array}$ & Bacillus sp. \\
\hline 14. & Modakkupatti & CBs4 & $\begin{array}{l}\text { Pale brown thick } \\
\text { slimy }\end{array}$ & $\begin{array}{l}\text { Light serrated } \\
\text { margin }\end{array}$ & Bacillus sp. \\
\hline 15. & Vazlavadi & CBs5 & $\begin{array}{l}\text { Pale brown } \\
\text { powdery slimy }\end{array}$ & Serrated margin & Bacillus sp. \\
\hline 16. & $\begin{array}{l}\text { Ramachadra } \\
\text { puram }\end{array}$ & CBs6 & Pale white slimy & $\begin{array}{l}\text { Wavy branched } \\
\text { margin }\end{array}$ & Bacillus sp. \\
\hline 17. & P.N.palayam & CBs7 & Pale white slimy & $\begin{array}{l}\text { Wavy branched } \\
\text { margin }\end{array}$ & Bacillus sp. \\
\hline 18. & Poolankinaru & CBs8 & Pale brown slimy & $\begin{array}{l}\text { Wavy branched } \\
\text { margin }\end{array}$ & Bacillus sp. \\
\hline 19. & Konnur & $\mathrm{CBs} 9$ & $\begin{array}{l}\text { Pale brown thick } \\
\text { slimy }\end{array}$ & $\begin{array}{l}\text { Wavy branched } \\
\text { margin }\end{array}$ & Bacillus sp. \\
\hline 20. & Pannaikinaru & CBs10 & Dull white slimy & Serrated margin & Bacillus sp. \\
\hline
\end{tabular}


Table.2 Identification and characterization of rhizobacteria from chickpea by biochemical characteristics

\begin{tabular}{|c|c|c|c|c|c|c|c|c|c|c|c|}
\hline \multirow{2}{*}{$\begin{array}{l}\text { S. } \\
\text { No }\end{array}$} & \multirow[t]{2}{*}{ Isolates } & \multicolumn{10}{|c|}{ Biochemical tests } \\
\hline & & $\begin{array}{l}\text { Gram's } \\
\text { Staining }\end{array}$ & $\begin{array}{c}\text { KOH } \\
\text { test }\end{array}$ & $\begin{array}{c}\text { HCN } \\
\text { production }\end{array}$ & $\begin{array}{c}\text { Catalase } \\
\text { test }\end{array}$ & $\begin{array}{c}\text { Starch } \\
\text { hydrolysis }\end{array}$ & $\begin{array}{c}\text { Gelatine } \\
\text { hydrolysis }\end{array}$ & $\begin{array}{c}\text { Growth } \\
\text { in } 7 \% \\
\mathrm{NaCl}\end{array}$ & $\begin{array}{c}\text { Citrate } \\
\text { utilization } \\
\text { test }\end{array}$ & $\begin{array}{l}\text { Siderophore } \\
\text { production }\end{array}$ & $\begin{array}{c}\text { Tentatively } \\
\text { identified as }\end{array}$ \\
\hline 1 & Pf1 & Pink & ++ & +++ & - & - & ++ & - & - & +++ & $\begin{array}{l}\text { Pseudomonas } \\
\text { fluorescens }\end{array}$ \\
\hline 2 & CPs 2 & Pink & ++ & + & - & - & ++ & - & - & + & Pseudomonas sp. \\
\hline 3 & CPs3 & Pink & ++ & + & - & - & ++ & - & - & +++ & Pseudomonas sp. \\
\hline 4 & CPs4 & Pink & ++ & + & - & - & ++ & - & - & + & Pseudomonas sp. \\
\hline 5 & CPs5 & Pink & ++ & ++ & - & - & ++ & - & - & + & Pseudomonas sp. \\
\hline 6 & CPs6 & Pink & ++ & + & - & - & ++ & - & - & + & Pseudomonas sp. \\
\hline 7 & CPs 7 & Pink & ++ & + & - & - & ++ & - & - & + & Pseudomonas sp. \\
\hline 8 & CPs8 & Pink & ++ & + & - & - & ++ & - & - & + & Pseudomonas sp. \\
\hline 9 & CPs9 & Pink & ++ & +++ & - & - & ++ & - & - & +++ & Pseudomonas sp. \\
\hline 10 & CPs10 & Pink & ++ & + & - & - & ++ & - & - & + & Pseudomonas sp. \\
\hline 11 & CBs 1 & Violet & - & - & ++ & ++ & - & + & ++ & - & Bacillus sp. \\
\hline 12 & $\mathrm{CBs} 2$ & Violet & - & - & ++ & ++ & - & + & + & - & Bacillus sp. \\
\hline 13 & CBs3 & Violet & - & - & ++ & ++ & - & + & ++ & - & Bacillus sp. \\
\hline 14 & CBs4 & Violet & - & - & + & ++ & - & + & + & - & Bacillus sp. \\
\hline 15 & CBs5 & Violet & - & - & ++ & ++ & - & + & ++ & - & Bacillus sp. \\
\hline 16 & CBs6 & Violet & - & - & ++ & ++ & - & + & + & - & Bacillus sp. \\
\hline 17 & CBs 7 & Violet & - & - & ++ & ++ & - & + & ++ & - & Bacillus sp. \\
\hline 18 & $\mathrm{CBs} 8$ & Violet & - & - & ++ & ++ & - & + & ++ & - & Bacillus sp \\
\hline 19 & CBs9 & Violet & - & - & + & ++ & - & + & + & - & Bacillus sp. \\
\hline 20 & CBs10 & Violet & - & - & ++ & ++ & - & + & + & - & Bacillus sp. \\
\hline
\end{tabular}

(+) - Positive; (-) - Negative; (++) - Medium production; (+++) - Strong production 
Table.3 Cultural and morphological characters of fungal antagonistic isolates of Trichoderma sp. from chickpea

\begin{tabular}{|c|c|c|c|c|c|}
\hline $\begin{array}{l}\text { S. } \\
\text { No }\end{array}$ & Locations & $\begin{array}{l}\text { Isolate } \\
\text { code }\end{array}$ & Cultural characters & $\begin{array}{l}\text { Morphological } \\
\text { characters }\end{array}$ & Referred as \\
\hline 1. & Coimbatore & $\begin{array}{c}\text { Tv1 } \\
\text { (TNAU) }\end{array}$ & $\begin{array}{l}\text { Dark greenish with } \\
\text { white fluffy growth }\end{array}$ & $\begin{array}{l}\text { Cylindrical } \\
\text { conidia }\end{array}$ & $\begin{array}{c}\text { Trichoderma } \\
\text { viride }\end{array}$ \\
\hline 2. & $\begin{array}{c}\text { Gomangalam } \\
\text { pudur }\end{array}$ & CTs1 & $\begin{array}{l}\text { White with greenish, } \\
\text { scattered growth }\end{array}$ & $\begin{array}{l}\text { Cylindrical } \\
\text { conidia }\end{array}$ & $\begin{array}{l}\text { Trichoderma } \\
\text { sp. }\end{array}$ \\
\hline 3. & Thippampatti & $\mathrm{CTs} 2$ & $\begin{array}{l}\text { Dark greenish adherent } \\
\text { growth }\end{array}$ & $\begin{array}{l}\text { Cylindrical } \\
\text { conidia }\end{array}$ & $\begin{array}{l}\text { Trichoderma } \\
\text { sp. }\end{array}$ \\
\hline 4. & Mukkonam & CTs3 & $\begin{array}{l}\text { Dark greenish adherent } \\
\text { growth }\end{array}$ & $\begin{array}{l}\text { Cylindrical } \\
\text { conidia }\end{array}$ & $\begin{array}{l}\text { Trichoderma } \\
\text { sp. }\end{array}$ \\
\hline 5. & Modakkupatti & CTs4 & $\begin{array}{l}\text { Dark greenish adherent } \\
\text { growth }\end{array}$ & $\begin{array}{l}\text { Cylindrical } \\
\text { conidia }\end{array}$ & $\begin{array}{l}\text { Trichoderma } \\
\text { sp. }\end{array}$ \\
\hline 6. & Valzavadi & CTs5 & $\begin{array}{l}\text { Dull green with white } \\
\text { adherent growth }\end{array}$ & $\begin{array}{l}\text { Cylindrical } \\
\text { conidia }\end{array}$ & $\begin{array}{l}\text { Trichoderma } \\
\text { sp. }\end{array}$ \\
\hline 7. & $\begin{array}{l}\text { Ramachadra } \\
\text { Puram }\end{array}$ & CTs6 & $\begin{array}{l}\text { White with greenish } \\
\text { fluffy growth }\end{array}$ & $\begin{array}{l}\text { Cylindrical } \\
\text { conidia }\end{array}$ & $\begin{array}{l}\text { Trichoderma } \\
\text { sp. }\end{array}$ \\
\hline 8. & P.N.Palayam & CTs7 & $\begin{array}{l}\text { Brownish adherent } \\
\text { growth }\end{array}$ & $\begin{array}{l}\text { Cylindrical } \\
\text { conidia }\end{array}$ & $\begin{array}{l}\text { Trichoderma } \\
\text { sp. }\end{array}$ \\
\hline 9. & Poolankinaru & CTs8 & $\begin{array}{l}\text { Greenish with white } \\
\text { fluffy growth }\end{array}$ & $\begin{array}{l}\text { Cylindrical } \\
\text { conidia }\end{array}$ & $\begin{array}{l}\text { Trichoderma } \\
\text { sp. }\end{array}$ \\
\hline 10. & Konnur & CTs9 & $\begin{array}{l}\text { Creamy white with } \\
\text { greenish fluffy growth }\end{array}$ & $\begin{array}{l}\text { Cylindrical } \\
\text { conidia }\end{array}$ & $\begin{array}{l}\text { Trichoderma } \\
\text { sp. }\end{array}$ \\
\hline
\end{tabular}

Table.4 Effect of different Pseudomonas sp. isolates against radial mycelial growth of Fusarium oxysporum f. sp. ciceris in vitro

\begin{tabular}{|c|c|c|c|c|}
\hline S. No. & Locations & Isolate No. & $\begin{array}{l}\text { Radial mycelial } \\
\text { growth (mm)* }\end{array}$ & $\begin{array}{l}\text { Percent inhibition } \\
\text { over control }(\%)\end{array}$ \\
\hline 1. & Gomangalampudur & CPs1 & $59.33^{e}$ & $34.07^{\mathbf{e}}$ \\
\hline 2. & Thippampatti & CPs2 & $52.67^{d}$ & $41.47^{\mathrm{d}}$ \\
\hline 3. & Mukkonam & CPs3 & $41.33^{\mathrm{a}}$ & $54.07^{\mathrm{a}}$ \\
\hline 4. & Modakkupatti & CPs4 & $77.33^{\mathrm{g}}$ & $14.07^{\mathrm{g}}$ \\
\hline 5. & Valzavadi & CPs5 & $66.00^{\mathrm{f}}$ & $26.66^{\mathrm{f}}$ \\
\hline 6. & $\begin{array}{l}\text { Ramachadra } \\
\text { Puram }\end{array}$ & CPs6 & $51.67^{d}$ & $42.58^{d}$ \\
\hline 7. & P.N.Palayam & CPs7 & $80.67^{\text {h }}$ & $10.36^{\mathrm{h}}$ \\
\hline 8. & Poolankinaru & CPs8 & $77.33^{\mathrm{g}}$ & $14.07^{\mathrm{g}}$ \\
\hline 9. & Konnur & CPs9 & $47.67^{c}$ & $47.03^{c}$ \\
\hline 10. & Coimbatore & Pf 1 & $43.67^{b}$ & $51.47^{\mathbf{b}}$ \\
\hline 11. & - & Control & $90.00^{\mathbf{i}}$ & $0.00^{i}$ \\
\hline
\end{tabular}

* Mean of the three replications

Means followed by a common letter are not significantly different at the $5 \%$ level by DMRT. 
Table.5 Effect of different isolates of Bacillus sp. against radial mycelial growth of Fusarium oxysporum f. sp. ciceris in vitro

\begin{tabular}{|c|c|c|c|c|}
\hline S. No. & Locations & Isolate No. & $\begin{array}{l}\text { Radial mycelial } \\
\text { growth }(\mathrm{mm}) *\end{array}$ & $\begin{array}{c}\text { Percent } \\
\text { inhibition } \\
\text { over control }(\%)\end{array}$ \\
\hline 1. & $\begin{array}{l}\text { Gomangalam } \\
\text { pudur }\end{array}$ & CBs 1 & 36.67 & $59.25^{b}$ \\
\hline 2. & Thippampatti & $\mathrm{CBs} 2$ & 55.67 & $38.14^{d}$ \\
\hline 3. & Mukkonam & $\mathrm{CBs} 3$ & 43.33 & $51.85^{\mathrm{c}}$ \\
\hline 4. & Modakkupatti & $\mathrm{CBs} 4$ & 58.67 & $34.81^{\mathrm{e}}$ \\
\hline 5. & Vazlavadi & CBs5 & 34.33 & $61.85^{\mathrm{a}}$ \\
\hline 6. & $\begin{array}{l}\text { Ramachadra } \\
\text { puram }\end{array}$ & CBs6 & 60.67 & $32.58^{\mathrm{e}}$ \\
\hline 7. & P.N.palayam & CBs7 & 56.33 & $37.41^{d}$ \\
\hline 8. & Poolankinaru & CBs8 & 63.33 & $29.63^{f}$ \\
\hline 9. & Konnur & $\mathrm{CBs} 9$ & 81.00 & $10.00^{h}$ \\
\hline 10. & Pannaikinaru & CBs 10 & 74.67 & $17.03^{\mathrm{g}}$ \\
\hline 11. & - & Control & 90.00 & $0.00^{\mathrm{i}}$ \\
\hline
\end{tabular}

*Mean of the three replications

Means followed by a common letter are not significantly different at the 5\% level by DMRT.

Table.6 Effect of different isolates of Trichoderma sp. isolates against radial mycelial growth of Fusarium oxysporum f. sp. ciceris in vitro

\begin{tabular}{|c|c|c|c|c|}
\hline S. No & Locations & Isolate. No & $\begin{array}{l}\text { Radial mycelial } \\
\text { growth (mm)* }\end{array}$ & $\begin{array}{l}\text { Per cent inhibition over } \\
\text { control }(\%)\end{array}$ \\
\hline 1. & Coimbatore & Tv1 & 29.00 & $67.77^{a}$ \\
\hline 2. & $\begin{array}{l}\text { Gomangalam } \\
\text { pudur }\end{array}$ & CTs1 & 55.33 & $38.52^{\mathrm{g}}$ \\
\hline 3. & Thippampatti & CTs2 & 34.00 & $62.22^{b}$ \\
\hline 4. & Mukkonam & CTs3 & 40.33 & $55.18^{d}$ \\
\hline 5. & Modakkupatti & CTs4 & 43.67 & $51.47^{\mathrm{e}}$ \\
\hline 6. & Valzavadi & CTs5 & 38.67 & $57.03^{d}$ \\
\hline 7. & $\begin{array}{l}\text { Ramachadra } \\
\text { Puram }\end{array}$ & CTs6 & 36.00 & $60.00^{c}$ \\
\hline 8. & P.N.Palayam & CTs7 & 51.33 & $42.96^{\mathbf{f}}$ \\
\hline 9. & Poolankinaru & CTs8 & 61.33 & $31.85^{\mathrm{h}}$ \\
\hline 10. & Konnur & CTs9 & 76.00 & $15.55^{\mathrm{i}}$ \\
\hline 11. & - & Control & 90.00 & $0.00^{\mathbf{j}}$ \\
\hline
\end{tabular}

*Mean of the three replications

Means followed by a common letter are not significantly different at the 5\% level by DMRT. 
Table.7 Effect of different fungicides against the radial mycelial growth of $F$. o. f. sp. ciceris (Foc 4) in vitro conditions

\begin{tabular}{|c|c|c|c|c|c|c|c|}
\hline \multirow{3}{*}{$\begin{array}{l}\text { S. } \\
\text { No }\end{array}$} & \multirow{3}{*}{ Fungicides } & \multicolumn{6}{|c|}{ Concentrations } \\
\hline & & \multicolumn{2}{|c|}{$0.025 \%$} & \multicolumn{2}{|c|}{$0.05 \%$} & \multicolumn{2}{|c|}{$0.1 \%$} \\
\hline & & $\begin{array}{c}\text { Radial } \\
\text { mycelial } \\
\text { growth } \\
(\mathrm{mm})^{*}\end{array}$ & $\begin{array}{c}\text { Percent } \\
\text { inhibition } \\
\text { over } \\
\text { control } \\
(\%)\end{array}$ & $\begin{array}{c}\text { Radial } \\
\text { mycelial } \\
\text { growth } \\
(\mathrm{mm}) *\end{array}$ & $\begin{array}{c}\text { Percent } \\
\text { inhibition } \\
\text { over } \\
\text { control } \\
(\%)\end{array}$ & $\begin{array}{c}\text { Radial } \\
\text { mycelial } \\
\text { growth } \\
(\mathrm{mm})^{*}\end{array}$ & $\begin{array}{c}\text { Percent } \\
\text { inhibition } \\
\text { over } \\
\text { control } \\
(\%)\end{array}$ \\
\hline 1. & $\begin{array}{c}\text { Carbendazim } \\
\text { (Bavistin) }\end{array}$ & 20.67 & $77.03^{a}$ & 18.33 & $79.63^{a}$ & 12.00 & $86.66^{\mathbf{a}}$ \\
\hline 2. & $\begin{array}{c}\mathrm{N}- \\
\text { trichloromethylthio- } \\
\text { 4-cyclohexane1,2- } \\
\text { dicarboximide } \\
\text { (Captan) }\end{array}$ & 38.00 & $57.77^{d}$ & 28.67 & $68.14^{\mathrm{c}}$ & 22.33 & $75.30^{d}$ \\
\hline 3. & $\begin{array}{l}\text { Copper oxychloride } \\
\text { (Kocide) }\end{array}$ & 52.33 & $41.85^{\mathrm{e}}$ & 33.00 & $63.33^{e}$ & 15.33 & $82.96^{\mathbf{b}}$ \\
\hline 4. & $\begin{array}{c}\text { Cymoxanil + } \\
\text { Mancozeb } \\
\text { (Curzate) }\end{array}$ & 71.00 & $21.11^{\mathrm{g}}$ & 40.67 & $54.81^{\mathrm{f}}$ & 28.33 & $68.52^{\mathrm{e}}$ \\
\hline 5. & $\begin{array}{c}\text { Fenamidone }+ \\
\text { Mancozeb (Sectin) }\end{array}$ & 85.33 & $5.18^{\mathrm{h}}$ & 54.33 & $39.63^{\mathrm{g}}$ & 30.33 & $66.30^{\mathrm{f}}$ \\
\hline 6. & $\begin{array}{c}\text { Fluopyram } \\
\text { (Luna Experience) }\end{array}$ & 33.00 & $63.33^{c}$ & 31.00 & $65.55^{\mathrm{d}}$ & 27.33 & $69.63^{e}$ \\
\hline 7. & $\begin{array}{c}\text { Iprovalicarb + } \\
\text { Probineb } \\
\text { (Melody Duo) }\end{array}$ & 59.00 & $34.44^{\mathrm{f}}$ & 58.00 & $35.55^{\mathrm{h}}$ & 32.33 & $64.07^{\mathrm{g}}$ \\
\hline 8. & $\begin{array}{l}\text { Isoprothiolane } \\
\text { (Fujione) }\end{array}$ & 24.33 & $72.96^{\mathbf{b}}$ & 21.67 & $75.92^{b}$ & 20.33 & $77.41^{\mathrm{c}}$ \\
\hline 9. & Control & 90.00 & $0.00^{\mathrm{i}}$ & 90.00 & $0.00^{\mathrm{i}}$ & 90.00 & $0.00^{h}$ \\
\hline
\end{tabular}

*Mean of the three replications.

Means followed by a common letter are not significantly different at the 5\% level by DMRT. 
Plate.1 In vitro screening of different isolates of Pseudomonas sp. on mycelial growth of $F$. oxysporum f. sp. ciceris

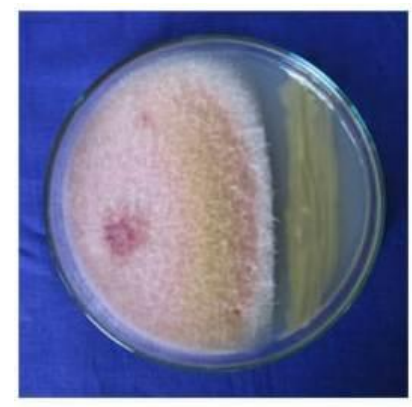

CPs. 1

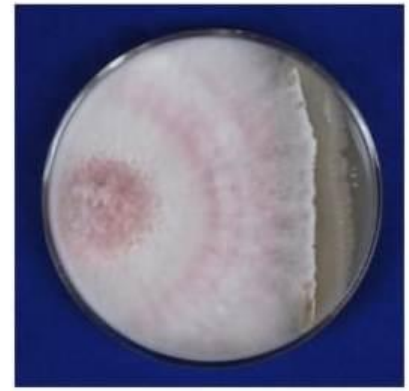

CPs. 4

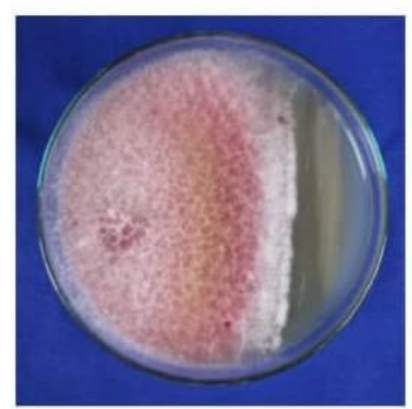

CPs. 7

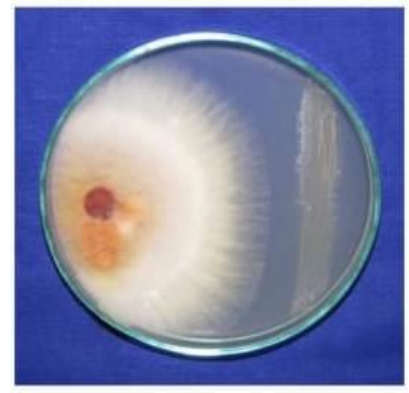

Pf 1

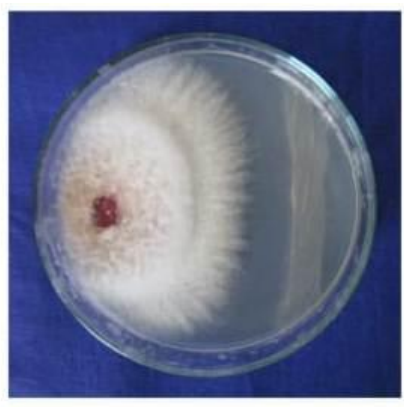

CPs. 2

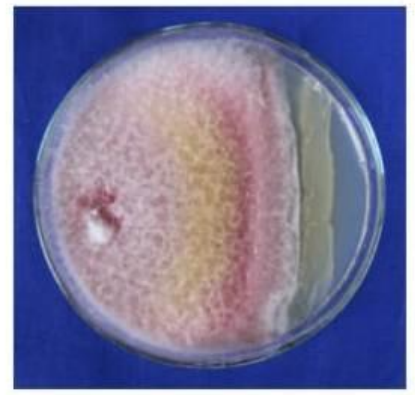

CPs. 5

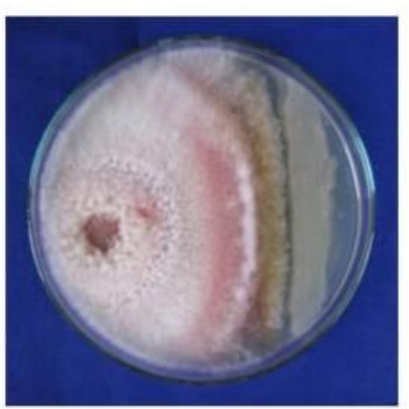

CPs. 8



Control

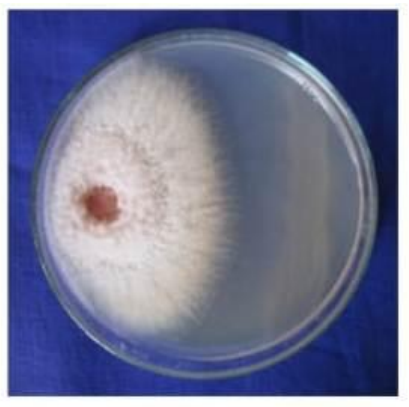

CPs. 3



CPs. 6

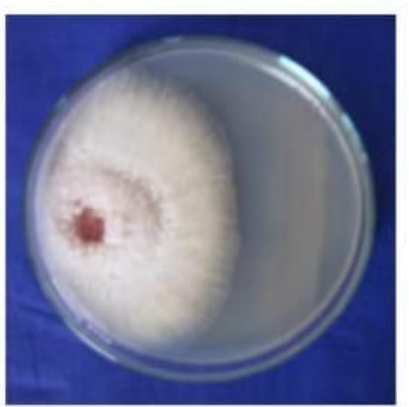

CPs. 9 
Plate.2 In vitro screening of different isolates of Bacillus sp. on mycelial growth of $F$. oxysporum f. sp. ciceris

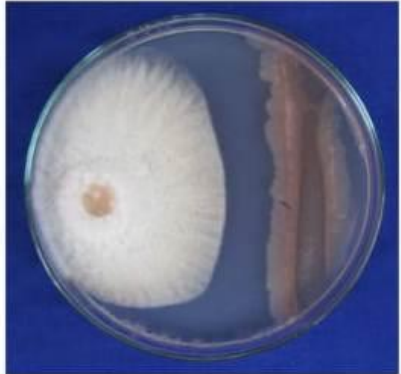

CBs. 1

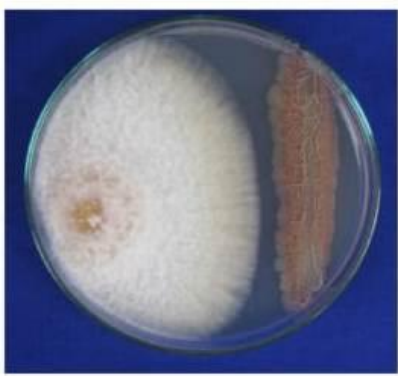

CBs. 4

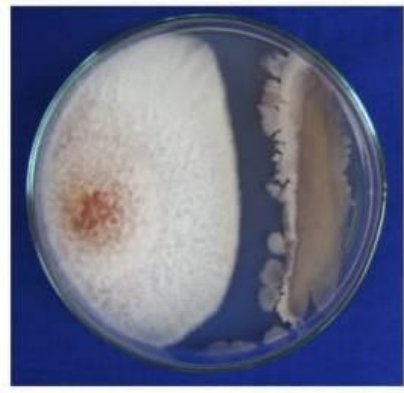

CBs. 7

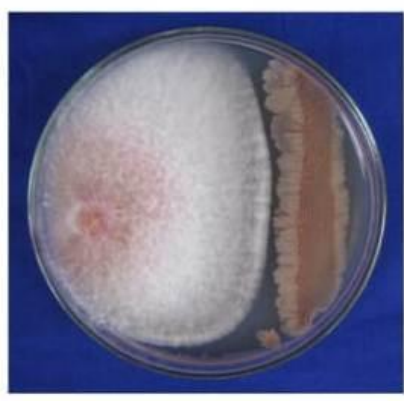

CBs. 10

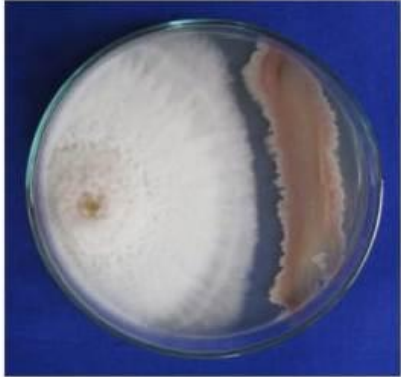

CBs. 2

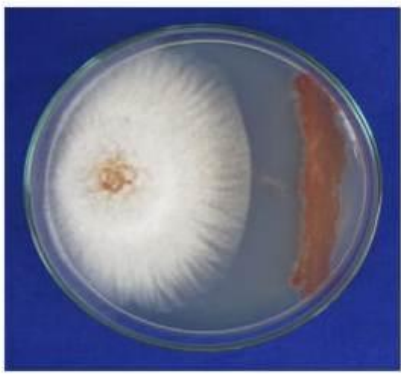

CBs. 5

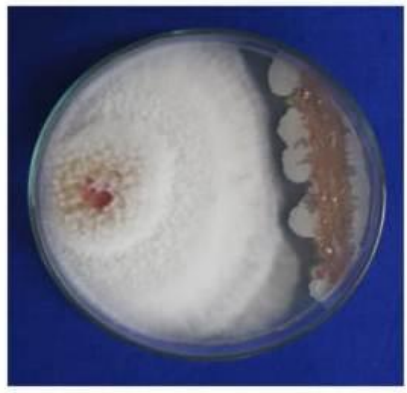

CBs. 8

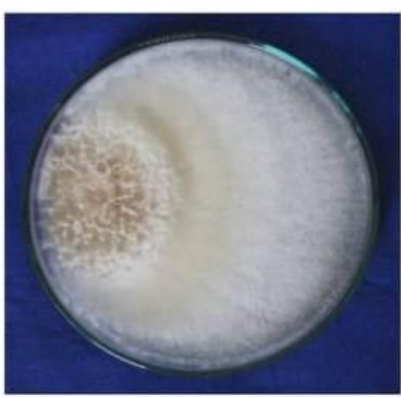

Control

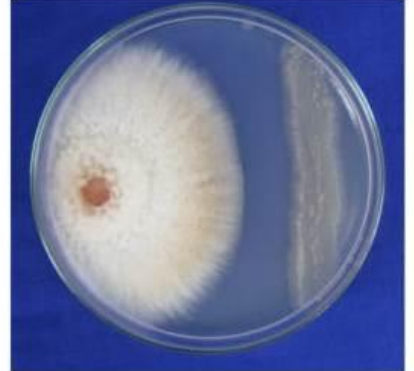

CBs. 3

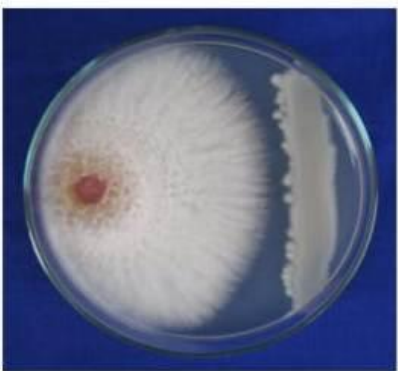

CBs. 6

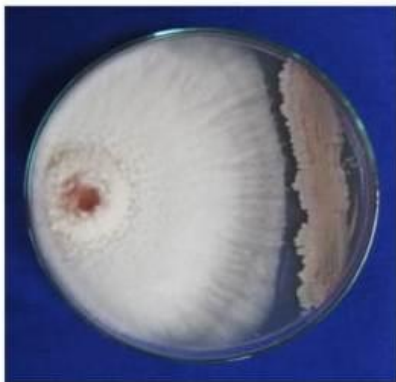

CBs. 9 
Plate.3 In vitro screening of different isolates of Trichoderma sp. on mycelial growth of $F$. oxysporum f. sp. ciceris

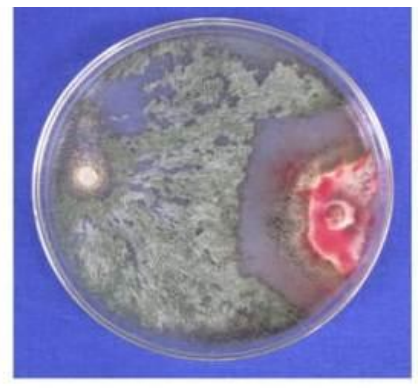

Tv 1



CTs. 3



CTs. 6

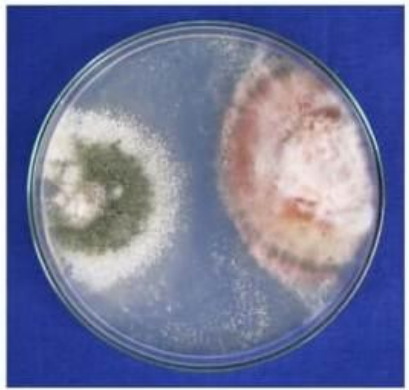

CTs. 9

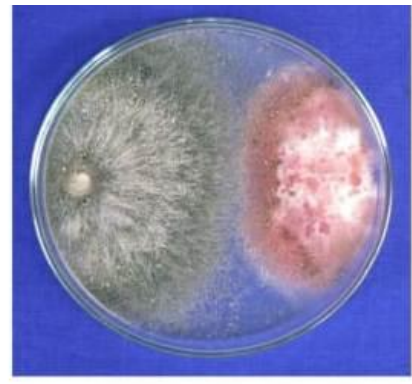

CTs. 1

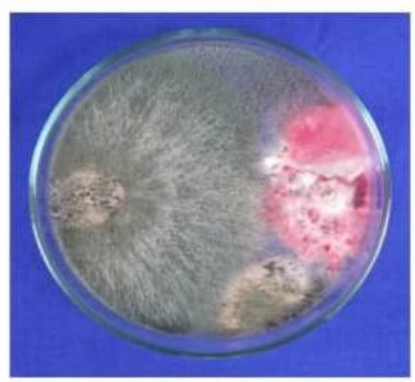

CTs. 4

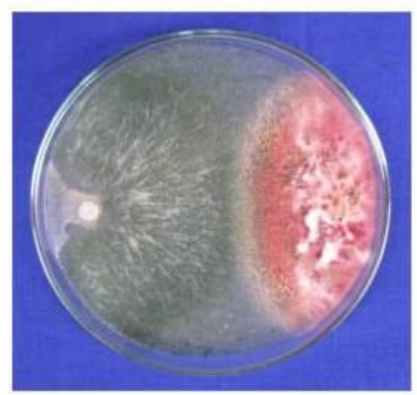

CTs. 7

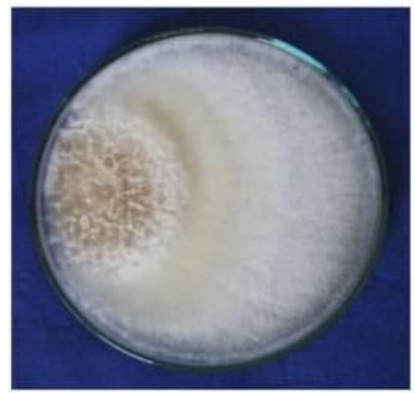

Control

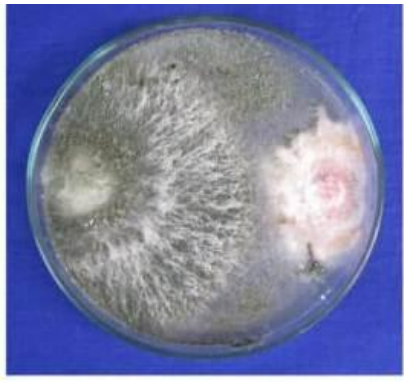

CTs. 2

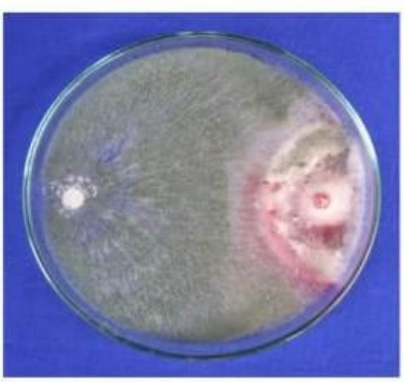

CTs. 5

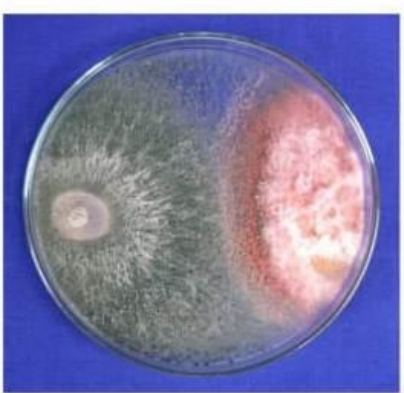

CTs. 8 
Plate.4 In vitro screening of different fungicides against on mycelial growth of $F$. oxysporum f. sp. ciceris

\section{$0.025 \%$}

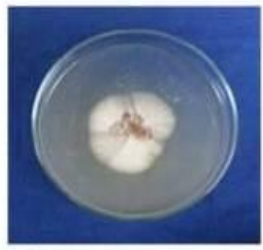

Carbendazim



Cymoxzanil+Mancozeb

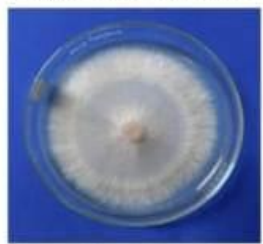

Iprovalicarb+Probineb

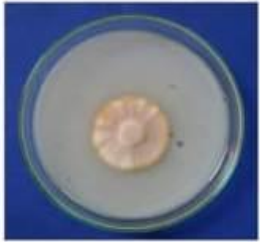

Captan

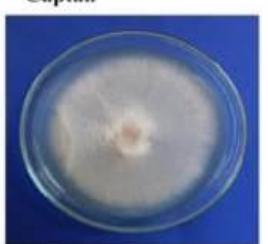

Fenamidone+Mancozeb

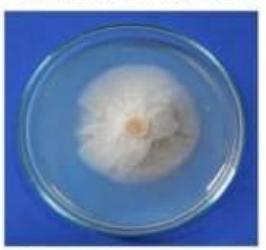

Isoprothiolane

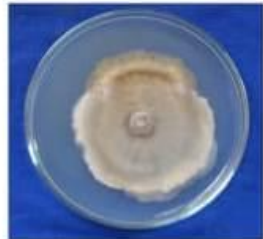

COC

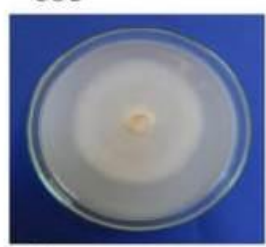

Fluopyram

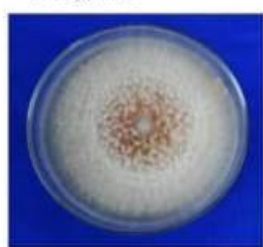

Control



Carbendazim

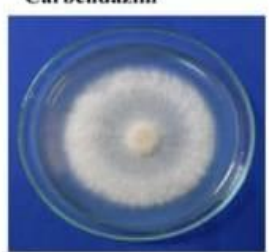

Cymoxzanil+Mancozeb

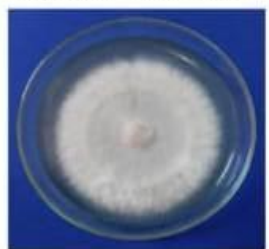

Iprovalicarb+Probineb

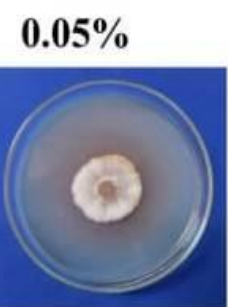

Captan

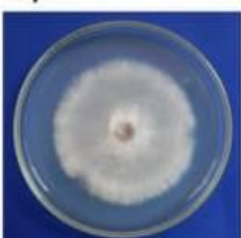

Fenamidone+Mancozeb

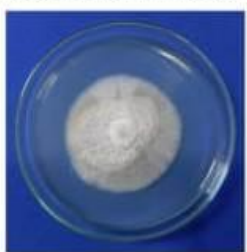

Isoprothiolane

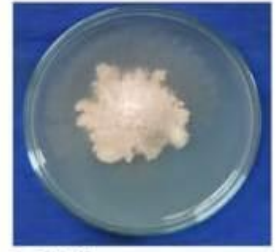

$\mathrm{COC}$

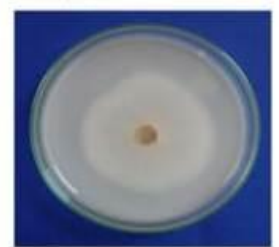

Fluopyram

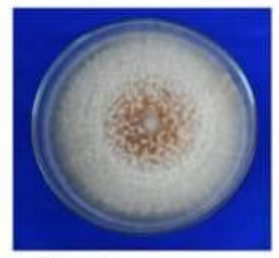

Control

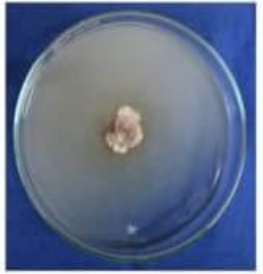

Carbendazim

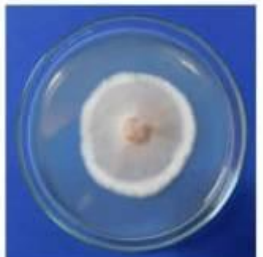

Cymoxzanil+Mancozeb

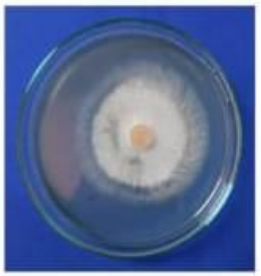

Iprovalicarb+Probineb

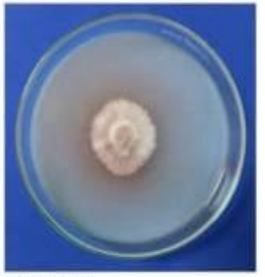

Captan

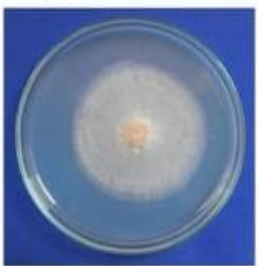

Fenamidone+Mancozeb

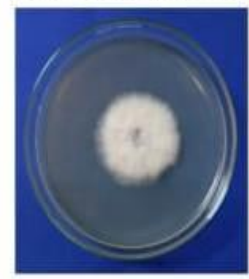

Isoprothiolane

$0.1 \%$

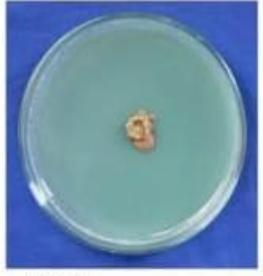

$\mathrm{COC}$

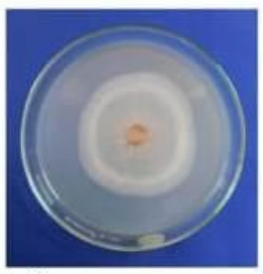

Fluopyram

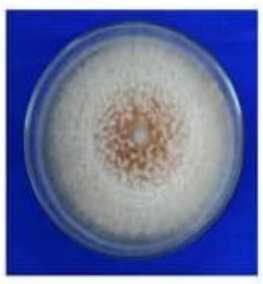

Control 
Inam-Ul-Haq et al., (2015) reported that three different species of rhizobacteria (RH-31, RH-32 and RH-33) among three isolates (RH33) $P$. psychrotolerans showed maximum inhibition against soil borne pathogens of $M$. phaseolina, $F$. oxysporum and $F$. solani.

\section{Bacillus sp.}

All the ten isolates of Bacillus sp., showed significant antagonistic activity of inhibition per cent from 10.00 to $61.85 \%$. Out of these isolates, CBs5 showed maximum inhibition per cent of $61.85 \%$ and followed CBs1 recorded $59.25 \%$ under in vitro conditions (Table 5; Plate 2). Karimi et al., (2012) reported that three isolates of Bacillus sp. (B28, B6 and B40) showed maximum mycelial growth inhibition per cent from 49.7 -51.2 against $F$. oxysporum f. sp. ciceris

\section{Trichoderma sp.}

In fungal antagonistic activity all the ten Trichoderma isolates were significantly inhibited mycelial growth from 15.55 to $67.77 \%$ against $F$. $o$. f. sp. ciceris. Out of these ten isolates, Tv1 showed maximum mycelial inhibition per cent of $67.77 \%$ followed by CTs3 at $62.22 \%$ inhibition under dual culture under in vitro (Table 6; Plate 3). Andrabi et al., (2011) reported that $T$. viride is exhibited superior antagonistic activity by maximum inhibition of mycelial growth per cent at $(86.21 \%)$ compared than $T$. virens $(85.29 \%)$ against $F$. oxysporum f. sp. ciceris.

\section{In vitro efficacy of fungicides}

Totally eight fungicides were used for checked the efficacy against $F$. oxysporum $\mathrm{f}$. sp. ciceris isolate Foc4. All the fungicides were significantly reduced the mycelial growth of pathogen. Among these eight fungicides, Carbendazim (Bavistin) showed maximum mycelial growth inhibition of 86.66 per cent and followed by Copper oxychloride (Kocide) at $82.96 \%$ at $0.1 \%$ concentration (Table 7; Plate 4). These results were similar to Maitlo et al., (2014) reported that screened with fourteen fungicides against with Foc the two fungicides carbendazim and thiophanatemethyl were highly effective at all concentrations of 1-10000ppm. Carbendazim showed maximum inhibition of mycelial growth at 100, 200 and $500 \mathrm{ppm}$ concentrations against $F$. oxysporum and $R$. solani under in vitro conditions (Andrabi et al., 2011)

\section{Acknowledgements}

Department of Pulses, Tamil Nadu Agricultural University, Coimbatore, India.

\section{References}

Ahmad, F., Ahmad, I. and Khan, M. S. 2008. Screening of free-living rhizospheric bacteria for their multiple growth promoting activities. Microbiol. Res., 163: 173-181.

Andrabi, M., Vaid, A. and Razdan, V. K. 2011. Evaluation of different measures to control wilt causing pathogens in chickpea. J. Plant Prot. Res., 51(1): 5559.

Cakmacki, R., Erat, M., Erdogan, U. and Donmez, M. F. 2007. The influence of plant growth -promoting rhizobacteria on growth and enzyme activities in wheat and spinach plant. J. Plant. Nutr Soil Sci., 170: 288-295.

Datta, M. R., Palit, C., Sengupta, K. M. and Banerjee, S. 2011. Plant growth promoting rhizobacteria enhance growth and yield of chilli (Capsicum annuum L.) under field conditions. Australian J. Crop Sci., 5: 531-536.

El-Katatany, M., Hetta, A., Silaban, G. and El-Komy, H. 2003. Improvement of cell wall degrading enzymes production by 
alginate encapsulated Trichoderma sp. Food Technol. Biotechnol., 41: 219225.

Govindarajan, M., Revathi, G. and Lakshminara-simhan, C. 2006. Improved yield of micro propagated sugarcane following inoculation by endophytic Burkholderia vietnamiensis. Pl. Soil, 280: 239-252.

Gyaneshwar, P., James, E. K., Mathan, N., Reddy, P. M., Reihol-Hurek, V. and Ladha, J. K. 2001. Endophytic colonization of rice by a diaztrophic strains of Serratia marcescens. J. Bacteriol., 183: 2634-2645.

Hossain, M. M., Hossain, N., Sultana, F., Naimul Islam, S. M., Shaikul Islam, M. and Alam Bhuiyan, M. K. 2013. Integrated management of Fusarium wilt of chickpea (Cicer arietinum L.) caused by Fusarium oxysporum f. sp. ciceris with microbial antagonist, botanical extract and fungicide. African J. Biotechnol., 12(29): 4699-4706.

Inam-Ul-Haq, M., Ibrahim Tahir, M., Hayat, R., Khalid, R., Ashfaq, M., Jamil, M., Naseem, S. and Ali, Z. 2015. Bioefficacy of rhizobacterial isolates against root infecting fungal pathogens of chickpea (Cicer arietinum L.). J. Plant Pathol. Microbiol., 3(011): 1-8.

Inam-Ul-Haq, M., Ibrahim Tahir, M., Hayat, R., Khalid, R., Ashfaq, M., Jamil, M., Naseem, S. and Ali, Z. 2015. Bioefficacy of rhizobacterial isolates against root infecting fungal pathogens of chickpea (Cicer arietinum L.). J. Plant Pathol. Microbiol., 3(011): 1-8.

Joseph, B., Patra, R. R. and Lawrence, R. 2007. Characterization of plant growth promoting rhizobacteria associated with chickpea (Cicer arietinum L.). Int J. Plant Prod., 1: 141-152.

Karimi, K., Amini, J., Harighi, B. and Bahramnejad, B. 2012. Evaluation of biocontrol potential of Pseudomonas and Bacillus spp. against Fusarium wilt of chickpea. Australian J. Crop Sci., 6(4): 695-703.

Khan, H. S. I., Saifulla, M., Nawaz, A. S. N., Somashekharappa, P. R. and Razvi, R. 2012. Efficacy of fungicides and biocontrol agents against Fusarium oxysporum f. sp. ciceris causing wilt of chickpea. J Environ. Ecol., 3: 570-572.

Khan, M. S., Zaidi, A, Wani, P. A., Ahemad, M. Oves, M. 2009. Functional diversity among Plant Growth-Promoting Rhizobacteria. Microbial strategies for Crop improvement., pp. 105-132.

Khilare, V. C., Ahmed, R., Chavan, S. S. and Kohire, O. D. 2009. Management of Fusarium oxysporum f. sp. ciceris by different fungicides. Bioinfolet., 6: 4143.

Kumar, P. and Mane, S. S. 2017. Efficacy and biocontrol agents against Fusarium oxysporum f. sp. ciceris. Int J. Current Microbiol. Appl Sci., 6(3): 1450-1455.

Kumari, S. and Khanna, V. 2014. Effect of antagonistic rhizobacteria coinoculated with Mesorhizobiam ciceris on control of Fusarium wilt in chickpea (Cicer arietinum L.). African J. of Microbiol Res., 8(1): 1255-1265.

Kumari, S. and Khanna, V. 2014. Effect of antagonistic rhizobacteria coinoculated with Mesorhizobiam ciceris on control of Fusarium wilt in chickpea (Cicer arietinum L.). African J. of Microbiol Res., 8(1): 1255-1265.

Landa, B. B., Hervas, A., Bettiol, W. and Jimenez-Diaz, R. M. 1997. Antagonistic activity of bacteria from the chickpea rhizosphere against Fusarium oxysporum f. sp. ciceris. Phytoparasitica., 25: 305-318.

Maitlo, S. A., Syed, R. N., Rustamani, M. A., Khuhro, R. D. and Lodhi, A. M. 2014. Comparative efficacy of different fungicides against Fusarium wilt of 
chickpea (Cicer arietinum L.). Pakistan J. Bot., 46(6): 2305-2312.

Navas-Cortes, J. A., Hau, B. and JimenezDiaz, R. M. 2000. Yield loss in chickpea in relation to development to Fusarium wilt epidemics. Phytopathol., 90: 1269-1278.

Nene, Y. L. and Reddy, M. V. 1987. Chickpea diseases and their control. Phytopathol., 42: 499-505.

Nene, Y. L., Sheila, V. K. and Sharma, S. B. 1984. A world list of chickpea (Cicer arietinum L.) and pigeonpea (Cajanus cajan L.) pathogens. Pulse progress report. ICRISAT, Patencheru. India. 32:19.

Poddar, R. K., Singh, D. V., and Dubey, S. C. 2004. Integrated application of Trichoderma harzianum mutant sand carbendazim to manage chickpea wilt (Fusarium oxysporum f. sp. ciceris). Indian J. Agrl Sci., 74(6): 346-348.

Schaad, N.W. 1992. Laboratory guide for identification of plant pathogen bacteria, 2nd Edn. International Book Distributing Co., Lucknow, pp. 44-58.

Sharma, K. D., Chenand, W., Muehbauer, F. J. 2004. A consensus set of differential lines for identifying races of Fusarium oxysporum f. sp. ciceris. Int. Chickpea and Pigeon pea NL., 11: 34-36.

Singh, J., Ratan, V. and Singh, N. 2009. Management of wilt of chickpea. Annals Plant Prot. Sci., 17: 248-249.

Subhani, M. N., Talib Sahi, S., Hussain, S, Ali, A., Iqbal, J. and Hammed, K. 2011. Evaluation of various fungicides for the control of gram wilt caused by Fusarium oxysporum f. sp. ciceris. African J. Agrl Res., 6(19): 4555-4559.

Thaware, D. S., Kohire, O. D. and Gholve, V. M. 2017. In vitro efficacy of fungal and bacterial antagonists against Fusarium oxysporum f. sp. ciceris causing chickpea wilt. Int J. Current Microbiol. Appl Sci., 6(1): 905-909.

\section{How to cite this article:}

Murali Sankar, P., S. Vanitha, A. Kamalakannan, P. Anantha Raju, P. Jeyakumar and Raguchander, T. 2018. Testing the Efficacy of Bio Control Agents and Fungicides against Fusarium oxysporum f. sp. ciceris under in vitro Conditions. Int.J.Curr.Microbiol.App.Sci. 7(06): 298-313. doi: https://doi.org/10.20546/ijcmas.2018.706.035 